\title{
Dependence of Nucleation Parameters for Polymer Crystallization from the Melt on Chemical Nature of a Macromolecule
}

\author{
Valery P. PRIVALKo \\ Institute of Macromolecular Chemistry, Academy of Sciences of the \\ Ukrainian SSR, 252160, Kiev 160, USSR.
}

(Received February 10, 1978)

\begin{abstract}
Analysis of experimental data on the temperature dependence of spherulitic growth rate from the melt for a number of linear, flexible-chain polymers has shown that the values of surface free energy of basal (fold-containing) face of a crystallization nucleus, $\sigma_{2}$, quantitatively correlate with polymer packing coefficient in the crystalline state, $K_{\mathrm{c}}$ in virtually the whole range of variation of these parameters known so far. Also the preexponential term, $G_{0}$, qunatitatively correlates with the "effective" free volume fraction, $\left(v_{\mathrm{a}}-v_{\mathrm{c}}\right) / v_{\mathrm{a}}$, where $v_{\mathrm{a}}$ and $v_{\mathrm{c}}$ are polymer specific volume in the amorphous and crystalline states, respectively. A tentative mechanism of crystalline phase nucleation and growth in supercooled polymer melts is proposed which takes account of density fluctuations in the melt.

KEY WORDS Nucleation/Chain Folding/Surface Free Energy / Packing Density / Free Volume Fraction / Density Fluctuations /
\end{abstract}

Temperature dependence of the crystalline phase growth rate from the isotropic melt is adequately accounted for by the classic biexponential equation ${ }^{1,2}$ :

$$
G=G_{0} \exp (-\Delta F / k T) \exp (-\Delta E / k T)
$$

where $\Delta F$ is the free energy of formation of a critical-size crystallization nucleus, $\Delta E$; the activation energy for a transport process across the meltcrystal interface; $G_{0}$, the preexponential coefficient; $k$, Boltzmann's constant; and $T$, the absolute temperature. For the case of a two-dimensional (surface) nucleation, $\Delta F$ is approximated, as ${ }^{2}$ :

$$
\Delta F=4 b_{0} \sigma_{1} \sigma_{2} T_{\mathrm{m}}{ }^{0} / \Delta H_{\mathrm{m}} \Delta T
$$

where $b_{0}$ is the thickness of a monomolecular crystalline layer on the growth face; $\sigma_{1}$ and $\sigma_{2}$, the (free) surface energies of lateral and basal faces of a crystallization nucleus; $T_{\mathrm{m}}{ }^{0}$, the "equilibrium" melting temperature; $\Delta H_{\mathrm{m}}$, the fusion enthalpy, and $\Delta T=T_{\mathrm{m}}{ }^{0}-T$, the degree of supercooling. Since the data on crystallization kinetics are generally found to obey eq 1 both for low-molecular weight, ${ }^{3}$ as well as polymeric ${ }^{2,3}$ liquids, the question arises, as to whether the nucleation parameters, $\sigma_{1}, \sigma_{2}$, and $G_{0}$, are the fundamental molecular char- acteristics of a substance, or only the arbitrary parameters of a corresponding theory. The conclusion on the validity of either opinion could have been obtained in principle by comparing experimental and theoretical values of the said parameters. However, so far such an approach (at least, based on semiempirical arguments) has been possible only for "monomeric" liquids. ${ }^{4,5}$ Thus, presently the only practical method to solve the formulated problem for polymers seems to be the establishment of a quantitative relationship between parameters $\sigma_{1}, \sigma_{2}$, and $G_{0}$ determined from the experiments on temperature dependence of crystallization rate from the melt, and the fundamental structural characteristics of a polymer found from independent measurements.

\section{Analysis of the Physical Meaning and Numerical Values of Parameters, $\sigma_{1}$ and $\sigma_{2}$ for Flexible- Chain Polymers}

We shall start by showing that the quantity, $\sigma_{2}$, for basal (i.e., fold-containing) faces of a crystallization nucleus, as obtained from kinetic data, generally should not coincide numerically with the parameter, $\sigma_{2}{ }^{\prime}$, for a "macroscopic" crystal, which enters the modified Tompson's equation ${ }^{2}$ 


\section{P. Privalko}

$$
T_{\mathrm{m}}=T_{\mathrm{m}}{ }^{0}\left(1-2 \sigma_{2}{ }^{\prime} / \Delta H_{\mathrm{m}} l\right)
$$

where $T_{\mathrm{m}}$ is the melting temperature of a foldedchain polymer crystal of the height, $l$. According to Gibbs' criterion, ${ }^{6}$ thermodynamically equilibrium crystal shape (at the constant volume, $V)$ must satisfy the condition,

$$
\sigma_{i} A_{i}=\text { const }
$$

where $\sigma_{i}$ and $A_{i}$ are surface free energy and the area of the $i$-th face respectively. As shown by Wulff, $^{7}$ the validity of condition (4) implies that there should exist a point inside the equilibrium crystal situated at a distance from $i$-th face $\left(P_{i}\right)$ proportional to $\sigma_{i}, i . e$.,

$$
\sigma_{1} / P_{1}=\sigma_{2} / P_{2}=\cdots=\sigma_{i} / P_{i}=\text { const }
$$

Taking into account that the height, $l^{*}$, and the width, $a^{*}$ of a critical-size crystallization nucleus in the polymer melt in the framework of kinetic theory, ${ }^{2}$ are $l^{*}=2 \sigma_{2} / \Delta F$ and $a^{*}=2 \sigma_{1} / \Delta F$, it is readily seen that the relation between $l^{*}$ and $a^{*}$ satisfies eq 5 , and consequently, the nucleus shape is the equilibrium one. On the other hand, after completion of crystallization, the lateral dimensions of lamellar crystals, as a rule, gradually become much larger than their heights, ${ }^{8}$ while the values of $\sigma_{2}{ }^{\prime}$ calculated from eq 3 either remain close to "kinetic" $\sigma_{2}{ }^{\prime} \mathrm{s}^{2,9}$, or significantly change. ${ }^{10-12}$ It follows therefrom that the lamellar morphology of crystallized polymers is essentially non-equilibrium, since for eq 5 to hold, the values of $\sigma_{1}$ would have been many times (in fact, tens-to-hundreds-fold) increased in comparison with the generally accepted $^{2}$ values. ${ }^{*}$ In other words, one may draw a broad conclusion that the structure of basal faces of crystallization nuclei and mature "macroscopic" crystals in bulk polymers (and, consequently, the numerical values of $\sigma_{2}$ and $\sigma_{2}{ }^{\prime}$ ) should be fundamentally different. The necessity of having to discriminate between $\sigma_{2}$ and $\sigma_{2}{ }^{\prime}$ was emphasized earlier by Mandelker, ${ }^{3,13}$ Kawai, ${ }^{14}$ Uhlmann, ${ }^{15}$ and others.

\footnotetext{
* Evidently, this argument will not work for the "mosaic" block model of Hosemann. ${ }^{18,19}$ However, as shown by recent experiments, ${ }^{20,21}$ the crystallographic coherence of polymeric crystals is conserved at distances of up to some thousands of Angstroms. This evidence apparently rules out the mosaic blocks concept, although this conclusion may be questioned. ${ }^{22}$
}

It is appropriate here to note that eq 4 and 5 are strictly applicable only to those crystals which have very large dimensions in comparison to the molecules from which they formed (i.e., such crystals should satisfy the definition of a "phase" ${ }^{16}$ ). In the case of "microscopic" nuclei of a new phase consisting of a limited number of molecules, the notion of crystalline "long"-range order becomes indefinite, and one is forced to consider only a "short"-range (i.e., quasicrystalline) order in the mutual arrangement of molecules of a nucleus. ${ }^{17}$ These arguments suggest that the empirical equations of Batchinsky-Macleod, ${ }^{23}$

$$
\sigma=\text { const }\left(\rho-\rho_{\mathrm{v}}\right)^{n}
$$

or Sugden ${ }^{24}$

$$
\sigma=\left[P\left(\rho-\rho_{\mathrm{v}}\right) / M\right]^{n}
$$

can be used as a first approximation to estimate the surface free energy not only of the melt $\left(\sigma_{\mathrm{a}}\right)$, but also of crystallization embrio $\left(\sigma_{\mathrm{c}}\right)$. In the above equations $\rho$ and $\rho_{\mathrm{v}}$ are densities of a substance in the condensed (liquid) and vapor sates respectively; $\boldsymbol{P}$ is a parachor, $M$ is molecular weight, and $n$ is an empirical parameter which, for the majority of low-molecular and polymeric liquids, has a value in the range of 3 to $4 .^{23-25}$ Writing the above equations for the melt and for crystallization nucleus respectively and assuming $\rho_{\mathrm{v}}=0$ and $n=4$, we obtain:

$$
\sigma_{\mathrm{c}}=\sigma_{\mathrm{a}}\left(\rho_{\mathrm{c}} / \rho_{\mathrm{a}}\right)^{4}
$$

Recently $\mathrm{Wu}^{26}$ obtained a similar equation.

We present in Table I the experimental values of $\sigma_{1}$ and $\sigma_{2}$, as well as other data necessary for applying eq 6 to many polymers. Values of $\sigma_{\mathrm{a}}$ and $\rho_{\mathrm{a}}$ were estimated from their temperature dependence plots ${ }^{25,26}$ at some arbitrarily chosen temperature, $T^{*}=0.9, T_{\mathrm{m}}$ being roughly midway in that temperature interval where the crystallization rates for most polymers were measured. We also used as $\rho_{\mathrm{c}}$ the tabulated values of density of a perfect crystal calculated from X-ray $\mathrm{data}^{27}$ and which were then extrapolated to $T^{*}$ on the assumption, $\mathrm{d} \ln \rho_{\mathrm{c}} / \mathrm{d} T=-2 \times 10^{-4} K^{-1}$. Pertinent experimental data for poly(tetrafluoroethylene) being unavailable, we used the values of $\rho_{\mathrm{a}}, \rho_{\mathrm{c}}$ and $\sigma_{\mathrm{a}}$ at $25^{\circ} \mathrm{C}$. To avoid any adverse effect of a specific nucleus model where it is a priori assumed $\sigma_{1} \neq$ $\sigma_{2},{ }^{2,3}$ we studied first the relation between the pro- 
Nucleation Parameters and Polymer Nature

Table I. Values of nucleation parameters and packing coefficients for linear polymers

\begin{tabular}{|c|c|c|c|c|c|c|c|c|c|c|}
\hline \multirow{2}{*}{ No. } & \multirow{2}{*}{ Polymer } & \multirow{2}{*}{$T_{\mathrm{m}}, \mathrm{K}$} & $\sigma_{1}$ & $\sigma_{2}$ & $\rho_{\mathrm{a}}$ & $\rho_{\mathrm{c}}$ & \multirow{2}{*}{$\begin{array}{c}\sigma_{\mathrm{a}}, \\
\mathrm{erg} / \mathrm{cm}^{2}\end{array}$} & \multirow{2}{*}{$\begin{array}{c}q, \\
\mathrm{kcal} / \mathrm{mol}\end{array}$} & \multirow[t]{2}{*}{$K_{\mathrm{c}}^{\mathrm{a}}$} & \multirow{2}{*}{$\begin{array}{l}A, \\
\AA^{2}\end{array}$} \\
\hline & & & \multicolumn{2}{|c|}{$\mathrm{erg} / \mathrm{cm}^{2}$} & \multicolumn{2}{|c|}{$\mathrm{g} / \mathrm{cc}$} & & & & \\
\hline 1 & Polyethylene & 418 & 12.7 & 75.0 & $\begin{array}{l}0.798 \\
0.856^{\mathrm{a}}\end{array}$ & $\begin{array}{l}0.985 \\
1.00^{\mathrm{a}}\end{array}$ & 29.1 & 3.94 & 0.735 & 18.2 \\
\hline 2 & Polypropylene (iso) & 449 & 8.8 & 44.5 & $\begin{array}{l}0.784 \\
0.856^{\mathrm{a}}\end{array}$ & $\begin{array}{l}0.905 \\
0.938^{\mathrm{a}}\end{array}$ & 24.8 & 4.4 & 0.700 & 34.3 \\
\hline 3 & Polystyrene (iso) & 514 & 4.2 & 32.0 & $\begin{array}{l}0.974 \\
1.112^{\mathrm{a}}\end{array}$ & $\begin{array}{l}1.018 \\
1.052^{\mathrm{a}}\end{array}$ & 28.8 & 6.5 & 0.705 & 70.5 \\
\hline 4 & Polybutene-1 (iso) & 403 & 7.2 & 15.5 & $0.860^{\circledR}$ & $0.886^{\mathrm{a}}$ & - & 1.7 & 0.655 & 60.6 \\
\hline 5 & $\begin{array}{l}\text { Poly(chlorotrifluoro- } \\
\text { ethylene) }\end{array}$ & 494 & 3.9 & 40.0 & $1.930^{\mathrm{a}}$ & $2.19^{\mathrm{a}}$ & - & 2.9 & 0.750 & 31.6 \\
\hline 6 & Poly(tetrafluoroethylene) & 607 & - & 250.0 & 一 & $2.40^{\mathrm{a}}$ & $25.2^{\mathrm{a}}$ & 11.0 & 0.825 & 28.2 \\
\hline 7 & Nylon 6 & 500 & 8.0 & 65.0 & $1.07^{\mathrm{a}}$ & $1.22^{\mathrm{a}}$ & - & 3.52 & 0.752 & 19.9 \\
\hline 8 & Nylon 66 & 545 & 8.5 & 45.0 & $1.07^{\mathrm{a}}$ & $1.22^{\mathrm{a}}$ & - & 2.65 & 0.752 & 19.9 \\
\hline 9 & Polyacrylonitrile $^{\mathrm{b}}$ & 590 & 5.1 & 151.0 & - & $1.27^{\mathrm{a}}$ & - & 13.3 & 0.780 & 27.7 \\
\hline 10 & Poly(dimethylsiloxane) & 233 & 5.2 & 4.5 & 1.132 & 1.06 & 25.6 & 1.2 & $0.605^{\mathrm{c}}$ & 58.0 \\
\hline 11 & Poly (propylene oxide) & 348 & 5.2 & 28.0 & $\begin{array}{l}0.954 \\
1.0^{\mathrm{a}}\end{array}$ & $\begin{array}{l}1.104 \\
1.102^{\mathrm{a}}\end{array}$ & 29.4 & 1.97 & 0.703 & 24.5 \\
\hline 12 & Poly(ethylene succinate) ${ }^{d}$ & 382 & 2.9 & 47.2 & - & $1.358^{\mathrm{a}}$ & - & 2.75 & 0.720 & 20.1 \\
\hline 13 & Poly(tetramethylene oxide) & 330 & 10.0 & $115.0^{\circ}$ & $\begin{array}{l}0.968 \\
0.980^{\mathrm{a}}\end{array}$ & $\begin{array}{l}1.136 \\
1.155^{\mathrm{a}}\end{array}$ & 40.7 & 6.4 & 0.753 & 19.3 \\
\hline 14 & Poly(ethylene oxide) & 348 & 13.0 & 41.5 & $\begin{array}{l}1.04 \\
1.13^{\mathrm{a}}\end{array}$ & $\begin{array}{l}1.286 \\
1.290^{\mathrm{a}}\end{array}$ & 41.4 & 2.57 & 0.723 & 21.5 \\
\hline 15 & Poly(ethylene adipate) & 343 & 7.5 & $45.2^{\mathrm{e}}$ & $\begin{array}{l}1.200 \\
1.205^{\mathrm{a}}\end{array}$ & $\begin{array}{l}1.335 \\
1.35^{\mathrm{a}}\end{array}$ & 45.6 & 2.6 & 0.745 & 19.9 \\
\hline 16 & Poly(methylene oxide) & 483 & 13.5 & 150.0 & $1.250^{\mathrm{a}}$ & $1.495^{\mathrm{a}}$ & - & 7.4 & 0.803 & 17.2 \\
\hline 17 & Selenium & 492 & 13.8 & 337.0 & $4.28^{\mathrm{a}}$ & $4.94^{\mathrm{a}}$ & - & 19.2 & 0.848 & 38.0 \\
\hline
\end{tabular}

a Values at $25^{\circ} \mathrm{C}$. b From ref 72. c From ref 40 . ' From ref 73 . ' From ref 59.

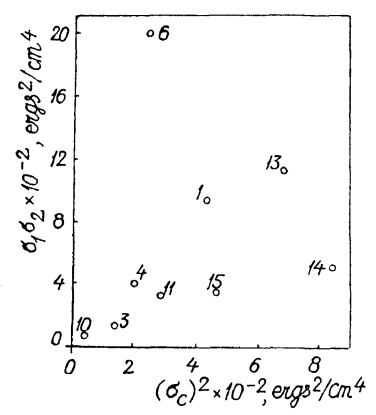

Figure 1. Relation between $\sigma_{1} \sigma_{2}$ and $\left(\sigma_{\mathrm{c}}\right)^{2}$. Throughout of this paper numbers at the data points refer to polymers listed in Table I.

duct, $\sigma_{1} \sigma_{2}$, and the quantity, $\left(\sigma_{\mathrm{c}}\right)^{2}$ (Figure 1). Exluding from consideration the data for polytetrafluoroethylene (point 6) and poly(ethylene oxide) (point 14), one can conclude that there is a trend for an increase in the product $\sigma_{1} \sigma_{2}$ with $\left(\sigma_{\mathrm{c}}\right)^{2}$. The same points also deviate from the trend line for other polymers on the plot, $\sigma_{2} v s . \sigma_{\mathrm{c}}$ (Figure 2),

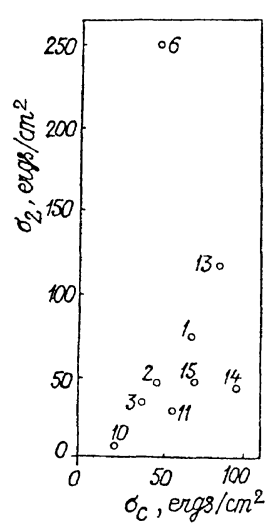

Figure 2. Relation between $\sigma_{2}$ and $\sigma_{\mathrm{c}}$.

while we observe a clear linear correlation between $\sigma_{\mathrm{c}}$ and $\sigma_{1}$ (Figure 3 ) which is described by equation

$$
\sigma_{1}=(0.15 \pm 0.03) \sigma_{\mathrm{c}}
$$

From the data obtained, it becomes evident that $\sigma_{2}$ and $\sigma_{1}$ are independent parameters, and this is contrary to Edward' opinion. ${ }^{28}$ This conclusion 


\section{P. Privalko}

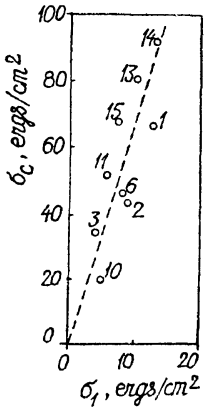

Figure 3. Relation between $\sigma_{\mathrm{c}}$ and $\sigma_{\mathbf{1}}$.

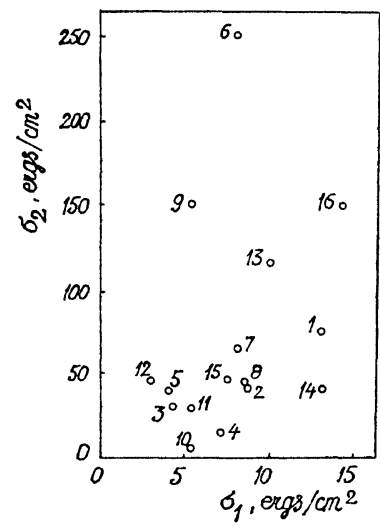

Figure 4. Relation between $\sigma_{2}$ and $\sigma_{1}$.

is supported by data in Figure 4.

Comparing eq 7 to Hoffman's equation ${ }^{2}$

$$
\sigma_{1}=\alpha b_{0} \Delta H_{\mathrm{m}}
$$

and taking account of eq 6 , we obtain (at $\alpha=0.1$ )

$$
\Delta H_{\mathrm{m}} / \sigma_{\mathrm{a}}=1.5 / b_{0}\left(\rho_{\mathrm{c}} / \rho_{\mathrm{a}}\right)^{4}
$$

The latter relation is similar to the one proposed by Hobin, ${ }^{29}$

$$
\Delta H_{\mathrm{coh}} / \sigma_{\mathrm{a}}=4 / b_{0}
$$

where $\Delta H_{\text {coh }}$ is the molar cohesion energy of an amorphous polymer. Solving eq 9 and 10 for $\sigma_{\mathrm{a}}$, we get

$$
\Delta H_{\mathrm{m}} / \Delta H_{\mathrm{coh}}=0.375\left(\rho_{\mathrm{c}} / \rho_{\mathrm{a}}\right)^{4}
$$

The dependence of tabulated ${ }^{30}$ values of $\Delta H_{\mathrm{m}}$ and $\Delta H_{\text {con }}$ is shown in Figure 5. The straight lines " $a$ " and " $b$ " in this figure were drawn according to eq 11 at $\rho_{\mathrm{c}} / \rho_{\mathrm{z}}=1.13^{30}$ and $\rho_{\mathrm{c}} / \rho_{\mathrm{z}}=1.09^{31}$ respectively. It can be seen that $\Delta H_{\mathrm{m}}$ increases

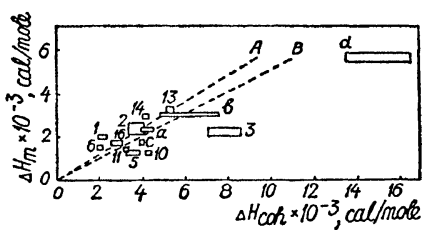

Figure 5. Relation between $\Delta H_{\mathrm{m}}$ and $\Delta H_{\mathrm{coh}}$. Lines "A" and "B" were calculated from eq 11 with $\rho_{\mathrm{c}} / \rho_{\mathrm{a}}$ $=1.3$ and 1.09 respectively: $a, 1,4$-polybutadiene; $b$, poly(vinyl chloride); c, 1, 4-polyisoprene; d, poly(ethylene terephthalate).

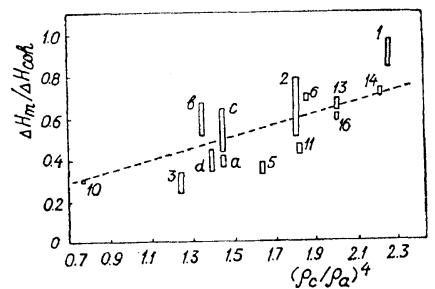

Figure 6. Relation between $\Delta H_{\mathrm{m}} / \Delta H_{\mathrm{coh}}$ and $\left(\rho_{\mathrm{c}}\right)$ $\left.\rho_{\mathrm{a}}\right)^{4}$

with $\Delta H_{\text {con }}$, although the lack of quantitative correlation between experimental values of these parameters and the "theoretical" lines " $a$ " and " $b$ " makes one to believe that the assumption, $\rho_{\mathrm{c}} / \rho_{\mathrm{z}}=$ const, ${ }^{30,31}$ never holds. A similar conclusion was arrived at on several occasions earlier..$^{32-34}$ As can be seen from the $\Delta H_{\mathrm{m}} / \Delta H_{\text {coh }} v s .\left(\rho_{\mathrm{c}} / \rho_{\mathrm{a}}\right)^{4}$ plot shown in Figures 6, a proper account of the dependence of the ratio, $\rho_{\mathrm{c}} / \rho_{\mathrm{a}}$, on the chemical nature of a macromolecule permits one to obtain a much improved agreement between the experimental data and theoretical predictions (broken line in Figure 6) based on eq 11. Especially gratifying here is the fact that the relation between $\Delta H_{\mathrm{m}} / \Delta H_{\mathrm{coh}}$ and $\rho_{\mathrm{c}} / \rho_{\mathrm{a}}$ for poly (dimethylsiloxane) which was postulated to have the ratio, $\rho_{\mathrm{c}} / \rho_{\mathrm{z}}$, below unity, ${ }^{32,33}$ is in a perfect quantitative agreement with eq 11 at $\rho_{\mathrm{c}} / \rho_{\mathrm{a}}=0.935$.

Thus, the preceding analysis has shown that the density increase due to the formation of a microscopic crystallization nucleus in the melt permits an understanding only of the numerical values of the surface free energy of the lateral faces of the nucleus with the aid of eq 6 , but to explain the nature of the surface free energy of the basal planes, one has to take into consideration the specificity of the chain-folding phenomenon. According to 
Hoffman, ${ }^{2,35}$ the value of $\sigma_{2}$ is directly related to the work, $q$, necessary for the formation of a single fold on the nucleus basal plane, through the following relation

$$
\sigma_{2}=(q / 2) A
$$

where $A$ is the chain cross-sectional area in the crystal. Numerical values of $A$ taken from ref 36, as well as values of $q$ calculated by eq 12 , are also included in table I. Analyzing the experimental values of $\sigma_{2}$ for polyethylene, Hoffman ${ }^{2,35}$ came to the conclusion that in the case of a regular (crystallographic) folding, $q$ may be approximated as $q=$ $n(\Delta \varepsilon)$, where $\Delta \varepsilon$ is the energy difference between the gauche- and trans-rotational isomers of the chain, and $n$ is the average number of gauchebonds in the fold. Unfortunately, this latter relation is of limited use for polymers in which structures are fundamentally different to polyethylene, since to determine $q$, one would need information both on the energy difference between rotational isomers, as well as on the number of higher-energy isomers in the fold, although this approach might be of use to solve the inverse problem: that is, to estimate $n$ from experimental values of $\sigma_{2}$ and $\Delta \varepsilon$. Attempting then to explain the variation of $\sigma_{2}$ for different polymers, Hof$\mathrm{fman}^{2,37}$ has also assumed that the stiffening of a molecular chain should be accompanied by a simultaneous increase of both $q$ and the melting temperature, $T_{\mathrm{m}}$. However, as can be seen from the corresponding plot in Figure 7 constructed from the data of Table I, no quantitative correlation between $q$ and $T_{\mathrm{m}}{ }^{0}$ exists. Therefore, it follows by necessity that to establish the dependence of $\sigma_{2}$ on the chemical nature of the chain repeating unit, some other approach should be attempted.

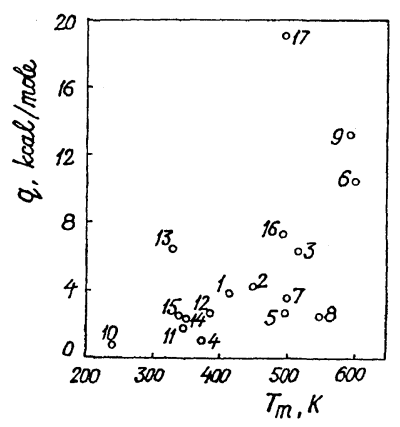

Figure 7. Relation between $q$ and $T_{\mathrm{m}}$.
A detailed analysis of energetics of formation of a single crystallographic fold in polyethylene single crystals ${ }^{2,38}$ has shown that the quantity, $\sigma_{2}$, may be expressed as the following sum:

$$
\sigma_{2}=\sigma_{1}+\sigma_{\mathbf{b}}+\sigma_{\mathrm{t}}
$$

where $\sigma_{\mathrm{b}}$ is the energy of chain bending by $180^{\circ}$ (i.e., the energy necessary to obtain the chain mirror image), and $\sigma_{\mathrm{t}}$ is the contribution of "twisting" energy required to twist thelchain into a nonparalle plane during its incorporation into crystalline lattice. Substituting experimental values $\sigma_{2}=75 \mathrm{erg} /$ $\mathrm{cm}^{2}$ and $\sigma_{1}=13 \mathrm{erg} / \mathrm{cm}^{2}$ for polyethylene ( $c f$. Table I) into eq 13 , one finds that the greatest (above $80 \%$ ) contribution to $\sigma_{2}$ is provided by the two terms, $\sigma_{\mathrm{b}}$ and $\sigma_{\mathrm{t}}$ which account for the energy of chain incorporation into the lattice. A similar trend is also evident for other polymers ( $c f$. Figure 4). Thus, if one assumes that the energy of chain incorporation into crystalline lattice varies in proportion to the packing density coefficient in the crystalline state, $K_{\mathrm{c}}$, then it is reasonable to expect the existence of a correlation between $\sigma_{2}$ and $K_{\mathrm{c}}$ for different polymers. ${ }^{39,40}$

The values of $K_{\mathrm{c}}$ in Table I for a number of flexible-chain polymers were taken from our previous work. ${ }^{36} K_{\mathrm{c}}$ for poly(dimethylsiloxane) was estimated by an empirical equation proposed earlier, ${ }^{36,39,40}$ since the numerical values of the Van der Waals volume for a chain repeating unit of this polymer so far were unavailable. ${ }^{41,42}$

Ordinary and semi-logarithmic plots of $\sigma_{2} v s$.

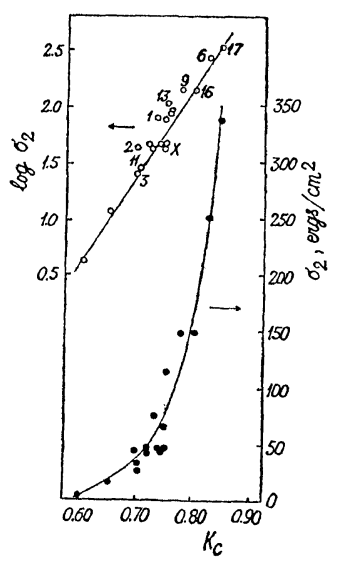

Figure 8. Relation of $\sigma_{2}$ and $\log \sigma_{2}$ on $K_{\mathrm{c}} ; X=5,7$, $12,14,15$. 


\section{P. Privalko}

$K_{\mathrm{c}}$ are shown in Figure 8. It can be readily seen that the values of $\sigma_{2}$ in the range of its variation encompassing about two orders of magnitude, are correlated to $K_{\mathrm{c}}$ through equation:

$$
\log \sigma_{2}\left(\mathrm{erg} / \mathrm{cm}^{2}\right)=7.65 K_{\mathrm{c}}-3.95
$$

at a standard deviation $\delta=0.12$. It is pertinent here to emphasize the following points. First, the data for polytetrafluoroethylene obey eq 14 which applies to flexible-chain polymers crystallizing by chain-folding mechanism. It follows therefrom that this polymer should also crystallize from the melt in folded-chain rather than in an extended-chain conformation ${ }^{* 43}$. Next, eq 14 also proved applicable for correlating the experimental data of an inorganic polymer, selenium, which is capable of chain-folded crystallization, too. $^{44,45}$ Taking $1.9 \AA$ as a Van der Waals radius of selenium, ${ }^{46}$ we calculated the intrinsic volume of the selenium chain repeating unit in the crystalline state as $69.6 \AA^{3}$ (this corresponds to three atoms in the unit cell). ${ }^{47}$ Using the values of crystallographic parameters for a hexagonal unit cell, $a=4.36 \AA$ and $c=4.96 \AA,{ }^{47}$ we obtain the unit-cell volume as $82 \AA^{3}$, which then yields $K_{\mathrm{c}}=$ $69.6 / 82=0.848$. Substituting the latter value into eq 14 we find $\sigma_{2}=355 \mathrm{erg} / \mathrm{cm}^{2}$, which is virtually identical to the experimental figure, $337 \mathrm{erg} / \mathrm{cm}^{2}{ }^{45}$ We may also note in passing that the latter value lies near the theoretically maximum value for folded-chain polymer crystals, since the limiting values of $\sigma_{2}$ from eq 14 for a system of flexible, constant cross-section rods turn out to be 115 $\mathrm{erg} / \mathrm{cm}^{2}$ for a cubic lattice $\left(K_{\mathrm{c}}=0.795\right)$ and 985 $\mathrm{erg} / \mathrm{cm}^{2}$ for hexagonal lattice $\left(K_{\mathrm{c}}=0.907\right)$. Finally, recalling that the value, $\sigma_{2}=4.5 \mathrm{erg} / \mathrm{cm}^{2}$, for poly(dimethylsiloxane) which probably has the minimal packing density in the crystalline state possible for flexible-chain polymers $\left(K_{\mathrm{c}}=0.605\right)$, also nicely conform to our equation 14 , one may generalize these findings by stating that the above correlation is applicable in the whole range of experimental values of $\sigma_{2}$ and $K_{\mathrm{c}}$ for such polymers.

\section{Analysis of the Preexponential Term, $G_{0}$}

In periodics, it is a common practice to discri-

* It is worth mentioning that poly(tetrafluoroethylene) was predicted 39,40 to have a "kinetic" value, $\sigma_{2}=$ 250 to $280 \mathrm{erg} / \mathrm{cm}^{2}$, two years before this value was obtained experimentally. ${ }^{12}$ minate between "fast-crystallizing" and "slowcrystallizing" polymers. To explain the drastic differences between crystallization rates from the melt encountered in practical work with different polymers, various empirical approaches were used, including the notion of differences in chain symmetry and/or flexibility, in melting temperatures, etc. $^{3,48}$ However, these criteria as such are of a very qualitative nature and have limited applicability. Turning now to eq 1 , one notes that during crystallization at low supercoolings (i.e., at $T_{\mathrm{m}}>$ $T \gg T_{\mathrm{g}}$ ) the quantity, $\Delta E$, may be roughly taken as constant for different polymers, thus making the major contribution to $G$ for the remaining terms, $\exp (-\Delta F / k T)$ and $G_{0}$. Therefore, at comparable $\Delta T$ 's and at $G_{0}=$ const $^{2}$ one should have expected the sudden drop in crystallization rate, $G$, with an increase of parameter $\sigma_{2}$ in the numerator of the first term in eq 1. However, experiments show an entirely opposite trend, ${ }^{49-50}$ namely, that it is those polymers having $\sigma_{2}$ of the order of 100 $\mathrm{erg} / \mathrm{cm}^{2}$ and above [i.e., polyethylene, poly (methylene oxide) and poly(tetrafluoroethylene)] which exhibit maximum $G$ 's ( $c f$. Table II). This observation suggests, therefore, that $G_{0} \neq$ const, and one should seek for an explanation of the extremely large differences in rates of crystallization for various polymers in numerical values. of preexponential term, $G_{0}$.

According to Hoffman's remark, ${ }^{2}$ unavoidable simplifications made in mathematical descriptions of a given phenomenon in the framework of a concrete molecular model, lead to very large discrepancies between experimental and theoretical values of $G_{0}$ (in fact, deviations as large as some orders of magnitude are not uncommon). The only theoretically sound attempt to explain the observed values of $G_{0}$ for polymers was undertaken so far by Hoffman, ${ }^{2,9}$ who assumed

$$
G_{0}=J_{0} b_{0}=b_{0} k T^{*} / h
$$

where $J_{0}=k T^{*} / h$ is the "standard" rate of nucleation in the melt, $T^{*}$ is the temperature of the maximum crystallization rate, and $h$ is the Planck's. constant. Values of $G_{0}$ for polyethylene and poly(chlorotrifluoroethylene) estimated from eq 15 deviate from the experimental ones by one to two orders of magnitude which was considered satisfactory. ${ }^{2,9}$ However, this discrepancy can be much more pronounced when the data for other 
Table II. Data obtained from measurements of spherulitic growth rate from the melt

\begin{tabular}{lccccccc}
\multicolumn{1}{c}{ Polymer } & $G^{\mathrm{a}}, \mathrm{min}$ & $T_{\mathrm{g}}, \mathrm{K}$ & $C_{2}, \mathrm{~K}$ & $G_{0}, \mathrm{~cm} / \mathrm{sec}$ & $\Delta S / k$ & \multicolumn{2}{c}{$\Delta S$, e.u. $\Delta S_{\mathrm{m}}$ e.u. } \\
\hline Polyethylene & $6.7(22)$ & 253 & 51.6 & $1.6 \times 10^{8}$ & 3.79 & 7.5 & 2.34 \\
& & 160 & & $10^{12}$ & & \\
Polypropylene & $7.0(49)$ & 258 & 75 & 700 & 0.98 & 1.95 & 2.2 \\
Polychlorotrifluoroethylene & $11.7(41)$ & 325 & 51.6 & $1.7 \times 10^{4}$ & 1.71 & 3.4 & 1.14 \\
Polystyrene & $0.15(50)$ & 358 & 75 & 15 & 0.46 & 0.91 & 1.95 \\
Nylon 6 & $14.4(46)$ & 335 & 110 & $2.43 \times 10^{6}$ & 2.08 & 4.15 & 1.4 \\
Nylon 66 & $14.4(46)$ & 335 & 110 & $1.55 \times 10^{4}$ & 2.08 & 4.13 & 1.55 \\
Poly(prypolene oxide) & $8.74(35)$ & 208 & 95 & 618 & 1.07 & 2.13 & 1.96 \\
Poly(ethylene succinate) ${ }^{\mathrm{b}}$ & - & - & - & 390 & 1.36 & 2.7 & 1.1 \\
Poly(ethylene adipate) & $15.8(35)$ & 220 & - & $1.05 \times 10^{4}$ & 1.36 & 2.7 & 1.26 \\
Poly(methylene oxide) & $1.25(48)$ & 190 & 51.6 & $1.05 \times 10^{11}$ & 4.47 & 8.92 & 3.0 \\
Selenium & $0.17(69)$ & 305 & 51.6 & $7.25 \times 10^{8}$ & 2.92 & 5.8 & 2.55 \\
\hline
\end{tabular}

a Values in parenthesis denote degree of supercooling $(\mathrm{K})$. b From ref 73.

polymers are taken into account. ${ }^{51}$ For example, eq 15 predicts that polymers with high $b_{0}$ 's and $T^{*}$, as compared with polyethylene (e.g., isotactic polystyrene), should have higher values of $G_{0}$, though in practice a completely different situation arises. ${ }^{51}$ This discrepancy clearly shows that the simple equation 15 cannot account for the specific structural peculiarities of the amorphous state of different polymers caused by the intrinsic differences of their chemical nature. Therefore, in place of eq 15 one should write a more general expression,

$$
G_{0}=G_{0}^{\prime} f(x)
$$

where the first term, $G_{0}{ }^{\prime}$, is defined by eq 15 , and the second, $f(x)$, defines the function of some structure-sensitive parameter, $x$. An equation of similar form was used recently by Lauritzen and Hoffman. $^{52}$

Some years ago, ${ }^{31,53,54}$ we formulated the concept of a "quasi-network" of short-range ordered regions in isotropic polymer melts arising from density fluctuations. In terms of this model, these regions of short-range, segmental order are the most likely candidates for crystallization nucleation in supercooled polymer melts. The general expression for the probability of density fluctuation in an isolated system has the following form $^{55}$ :

$$
\mathrm{d} w(\rho)=f(\rho) \mathrm{d} \rho
$$

where $f(\rho)=A \exp (\Delta S / k)$ is the probability distribution density of parameter $\rho, \Delta S$ is the entropy difference between the given (i.e., instantaneous), and the equilibrium (i.e., time-averaged) states of the system, and $A$ is a constant. It can be reasonably assumed that the number of "primary" crystallization nuclei in the melt at a given temperature which forms per unit time due to density fluctuations (in other words, the nucleation rate responsible for numerical value of $G_{0}$ ), will depend on the probability distribution density, $f(\rho)$, that is

$$
G_{0}=f(\rho) \text { and } G_{0}{ }^{\prime} f(x)=A \exp (\Delta S / k)
$$

Setting $G_{0}{ }^{\prime}=$ const $=A$, we obtain

$$
f(x)=\exp (\Delta S / k)
$$

The latter expression is similar in form to Eyring's definition of activation entropy for transport processes as a measure of deviation between experimental values of a preexponential term and theoretical ones calculated from simple models. ${ }^{56}$ However, as follows from the above discussion, the quantity, $\Delta S$, in eq 19 has the physical meaning of difference between entropies of a system in nonequilibrium and equilibrium states, respectively. Assuming now that the non-equilibrium state corresponds to the one in which all segments enter the quasi-crystalline regions of a short-range order, and that the equilibrium state is that of an isotropic melt with homogeneous segmental distribution, and using the general definition of entropy as a measure of order, we come finally to the conclusion that $\Delta S$ should be a function of a dimensionless ratio, $\left(v_{\mathrm{a}}-v_{\mathrm{c}}\right) / v_{\mathrm{a}}$, where $v_{\mathrm{a}}=1 / \rho_{\mathrm{a}}$ and $v_{\mathrm{c}}=1 / \rho_{\mathrm{c}}$ are 


\section{P. Privalko}

polymer specific volumes in the amorphous and crystalline states.

The values of $G_{0}$ for a number of polymers calculated from experimental data on the temperature dependence of spherulitic growth rates from the melt ${ }^{2,37,49,51,57,58}$ are listed in Table II. Since the numerical values of $G_{0}$ are very sensitive to the analytic form of an approximation used to define $\Delta E$, we have employed in the calculations the Hoffman's relation, ${ }^{2}$

$$
\Delta E=4120 T /\left(C+T-T_{\mathrm{g}}\right)
$$

with variable parameter $C$. Values of the ratio, $\left(v_{\mathrm{a}}-v_{\mathrm{c}}\right) / v_{\mathrm{a}}$, at $25^{\circ} \mathrm{C}$, were evaluated from the data in Table I.

Plots of $\log G_{0}$ and $\log \left(\log G_{0}\right)$ vs. $\left(v_{\mathrm{a}}-v_{\mathrm{c}}\right) / v_{\mathrm{a}}$

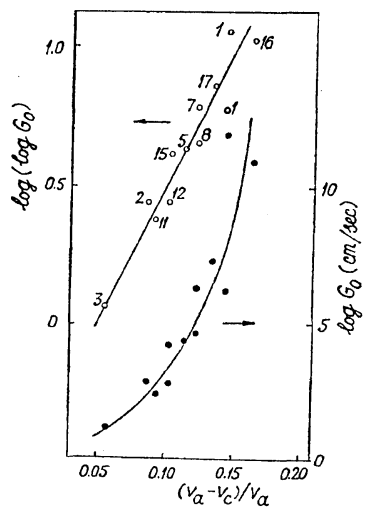

Figure 9. Dependence of $\log G_{0}$ and $\log \left(\log G_{0}\right)$ on $\left(v_{\mathrm{a}}-v_{\mathrm{c}}\right) / v_{\mathrm{a}}$.

are shown in Figure 9. The data in Figure 9 are adequately accounted for by equation (solid line in Figure 9):

$$
\log \left(\log G_{0}\right)(\mathrm{cm} / \mathrm{sec})=-0.5+10\left(v_{\mathrm{a}}-v_{\mathrm{c}}\right) / v_{\mathrm{a}}
$$

at a standard deviation $\delta=9.95 \times 10^{-2}$. Comparing eq 17,18 , and 19 and setting $G_{0}{ }^{\prime}=$ const $=$ $5 \times 10^{5} \mathrm{~cm} / \mathrm{sec}$ for all polymers, ${ }^{*}$ we $\operatorname{get}^{59}$ :

$$
\Delta S / k=-0.89+10\left(v_{\mathrm{a}}-v_{\mathrm{a}}\right) / v_{\mathrm{c}}
$$

The numerical values of the ratio, $\Delta S / k$, calculated

* This value corresponds to "statistically-average" values $b_{0}=6 \AA$ and $T^{*}=400 \mathrm{~K}$. Errors brought about by deviations of experimental values of these parameters from "statistically-average" ones by 30 $50 \%$, remain within the limits of standard deviation.

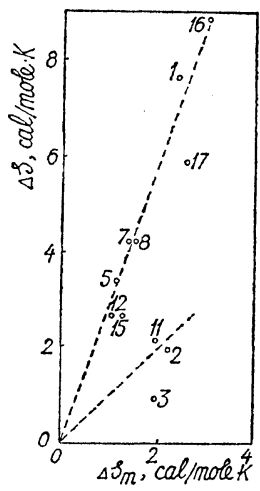

Figure 10. Relation between $\Delta S$ and $\Delta S_{\mathrm{m}}$.

by eq 21 , are also listed in Table II. Unfortunately, it is rather difficult now to attribute any definite physical significance to these values since this would require an a priori guess as to the dimensions of structural "growth segments" deposited onto the crystal growth face. However, we have tried to find a correlation between the values of $\Delta S$ per mole of "growth segments" as calculated from the ratios, $\Delta S / k$, in Table II, and those of the fusion entropy per mole of main-chain bonds, $\Delta S_{\mathrm{m}}{ }^{3}$. The resulting plot is shown in Figure 10 from which it becomes clear that the studied polymers can be subdivided into two groups, the first of which obeys the relation,

$$
\Delta S \cong 3 \Delta S_{\mathrm{m}}
$$

while for the second,

$$
\Delta S=\Delta S_{\mathrm{m}}
$$

seems to hold. Interestingly, the first group consists of polymers with a rather simple, symmetrical molecular structure (e.g., aliphatic polyethers, polyesters, polyamides, and the like), for which the inequality, $v_{\mathrm{c}} / v_{\mathrm{a}}<0.9$, is usually observed; but, for the remaining three polymers of the second group [polypropylene, poly(propylene oxide) and polystyrene] with massive side substituents which crystallize in helical conformation, the ratio $v_{\mathrm{c}} / v_{\mathrm{a}}$ axceeds 0.9 . We may recall here that splitting of the plots describing the dependence of various polymer properties on conformational characteristics of macromolecules having different conformations in the crystalline state, is not a rare incident. ${ }^{60,61}$ These results cause one to believe 
that for polymers of the first group having a higher values of $G_{0}$ and obeying eq 22 , the dimensions of "growth segments" should be substantially greater than the three main-chain bonds, while for the second group polymers which have lower values of $G_{0}$, the crystal growth should proceed through transport of "growth segments" comparable to the single bonds of the main chain. These arguments compare favorably to the predictions of Lauritzen and Hoffman ${ }^{52}$ on the existence of two mechanisms of polymer crystallization. The first of these is characterized by larger values of $G_{0}$ and corresponds to continuous deposition of long individual "growth segments" onto the crystal growth face. But during crystallization by a second mechanism (i.e., that with lower $G_{0}$ ), the growth proceeds through a simultaneous deposition of many small "growth segments" on the growth plane and their subsequent slow "incorporation" into crystalline lattice. It should be mentioned here that polyethylene which enters our first group of polymers, crystallizes by a first mechanism, while the polymer from the second group, isotactic polystyrene, obeys the second crystallization mechanism. ${ }^{52}$

The above results might be given the following tentative molecular interpretation. Density fluctuations in supercooled polymer melts are "frozen-in" at temperatures where the bulk free energy loss, due to the formation of crystallization nuclei, over-compensates the free energy gain due to formation of the melt-crystal interface. The subsequent process of the "growth segments" deposition on the nucleus growth face will proceed faster in polymers from the first group having higher values of the "effective" free volume fraction, $\left(v_{\mathbf{a}}-v_{\mathbf{c}}\right) / v_{\mathbf{a}}$. However, a similar process in the second-group polymers is expected to be much slower. These arguments are in a good qualitative correspondence to the proposed mechanism of transport processes in microheterogeneous melts of flexible-chain polymers. ${ }^{62}$

\section{CONCLUSIONS}

The results of our analysis have shown that the experimental values of the surface free energy of basal (fold-containing) planes of a crystallization nucleus, $\sigma_{2}$, quantitatively correlate with the polymer packing coefficient in the crystalline state,
$K_{\mathrm{c}}$, in practically the whole range of variation of these parameters for flexible-chain polymers. This result thus confirms our suggestion that the experimental values of $\sigma_{2}$ reflect mainly the contribution of a work required to incorporate crystallographically the chain "growth segments" into a crystalline lattice. Moreover, this quantitative correlation may serve as a practical tool of correct assessment of experimental data. For example, analyzing the conflicting values of $\sigma_{2}$ for poly(vinylidene fluoride), Mancarelly and Martuscelli $^{63}$ select as the more probable and the lower figure, $65 \mathrm{erg} / \mathrm{cm}^{2}$. However, since this polymer has $K_{\mathrm{c}}$ at least comparable to that for poly(tetrafouroethylene), ${ }^{36}$ it turns out that higher values, 239 to $349 \mathrm{erg} / \mathrm{cm}^{2}{ }^{63}$ are more appropriate here. Our analysis of Martuscelli's data on the kinetics of isothermal crystallization from the melt of copolymers of dienes and polyesters ${ }^{64}$ has also shown that the regular decrease of $\sigma_{2}$ accompanying the increase of the double-bonds content in the chain can be quantitatively accounted for with our eq 14 since the concomitant increase in the lateral dimensions of the crystalline unit cell (at the constant periodicity along the chain axis) leads directly to lower $K_{\mathrm{c}}$ 's.

Quantitative analysis of the preexponential term, $G_{0}$, for a number of polymers revealed the inadequacy of existing theoretical estimates of this parameter and formed the basis for suggestion, that $G_{0}$ should depend on the probability of a segmental density fluctuation in polymer melts expressed empirically as the dimensionless ratio, $\left(v_{\mathrm{a}}-v_{\mathrm{c}}\right) / v_{\mathrm{a}}$. This suggestion was confirmed by a quantitative correlation between $G_{0}$ and the "effective" free volume fraction, $\left(v_{\mathrm{a}}-v_{\mathrm{c}}\right) / v_{\mathrm{a}}$, in a very large (in fact, encompassing about ten orders of magnitude) range of variation of experimental values of $G_{0}$ for spherulitic growth rates from the melt. By disussing this correlation in terms of a classical definition of fluctuation probability as of a function of an entropy difference, $\Delta S$, between equilibrium and non-equilibrium states of an isolated system (that is, isotropic polymer melt), it has been shown that dependence of $\Delta S$ on fusion entropy per main-chain bond is markedly different for polymers of a simple molecular structure having $v_{\mathrm{c}} / v_{\mathrm{a}}$ below about 0.9 , on one hand, and for polymers with bulky side substituents and $v_{\mathfrak{c}} / v_{\mathrm{a}}$ above 0.9 , on the other hand. This difference is 


\section{P. Privalko}

consistent with the concept that the dimensions of the "growth segments" for the fisrt group of polymers should be substantially greater than those for the second group. It also significant that the peculiar feature of melt crystallization for polymers from the first group [polyethylene, poly(ethylene oxide), poly (tetramethylene oxide), etc.] is a very pronounced process of "secondary" crystallization proceeding primarily via mechanism of "thickening" of lamellar crystals accompanied by the "smoothing" of their basal planes. ${ }^{65-69}$ For polymers from the second group (polystyrene, polybutene-1, polypropylene, and the other helicalchain polymers) the phenomenon of secondary crystallization is either entirely absent, ${ }^{70}$ or very weak, the lamellar height being essentially unchanged. ${ }^{71}$

On the basis of these observations, it is tempting to speculate on the qualitative mechanism of polymer crystallization from the melt. Density fluctuations in supercooled polymer melts bring about the formation of a quasinetwork of ordered regions serving as potential nuclei for crystalline phase. The "freezing-in" of fluctuations (i.e., appearance of stable crystallization nuclei) will take place under conditions of the energy losses during formation of a melt-crystal interface. This depends mainly on the surface free energy, $\sigma_{2}$, becoming less than the loss of free energy of the bulk polymer due to formation of thermodynamically stable crystalline nuclei. For polymers of the first group possessing higher values of $K_{\mathrm{c}}$, the process of incorporation of long growth segments into denselypacked crystalline lattice will require higher energy for the deformation of the latter. This is reflected in the enhanced values of $\sigma_{2}$, while the energy (and, correspondingly, $\sigma_{2}$ ) required for a similar process in loosely-packed polymers of the second group, should be considerably lower. Moreover, rather large values of the free volume fraction, $\left(v_{\mathrm{a}}\right.$ $\left.-v_{\mathrm{c}}\right) / v_{\mathrm{a}}$, for polymers of the first group are the likely prerequisites for significant fluctuations of the period of chain folding (that is, dimensions of the growth segments) during the initial period of crystallization, and their smoothing-out during the final (secondary) crystallization stage. Such processes should be much less pronounced in polymers of the second group having lower values of the ratio, $\left(v_{\mathrm{a}}-v_{\mathrm{c}}\right) / v_{\mathrm{a}}$. This picture is in qualitative agreement both with the available experimental data, ${ }^{65-71}$ as well as with the predictions of a more sophisticated molecular theory. ${ }^{52}$

\section{Note Added in Proof}

Recently Miller and Boyer (J. Polym. Sci., Polym. Phys. Ed., 16, 371 (1978)) obtained the following relationship between $\sigma_{2}$ and the chain crosssectional area $A$

$$
\log \sigma_{2}=k_{1}-k_{2} \log A
$$

where $k_{1}$ and $k_{2}$ are empirical constants for different polymer families, the latter being very close to unity. This equation is in quallitative accord with eq 14 of the present paper in view of a rough correlation between $K_{\mathrm{c}}$ and $A$ (V. P. Privalko, Polym. J., 7, 202 (1975)). Moreover, inserting eq 8 of this paper into expression, $K_{\mathrm{g}}=4 b_{0} \sigma_{1} \sigma_{2} T_{\mathrm{m}} /$ $k \Delta H_{\mathrm{m}}$, and taking logarithms, one obtains equation identical to that of Miller and Boyer, viz.,

$$
\log \sigma_{2}=k_{1}{ }^{\prime}-k_{2}{ }^{\prime} \log A
$$

where $k_{1}{ }^{\prime}=\log K_{\mathrm{g}}-\log T_{\mathrm{m}}-\log (4 \alpha / k), k_{2}{ }^{\prime}=1$ and $A=b_{0}{ }^{2}$. Therefore, to estimate $\sigma_{2}$ using this approach one needs either knowing the numerical value of $K_{\mathrm{g}}$ which can not be made on an a priori basis, or making a guess on to what polymer family a given polymer belongs in order to choose the empirical value of $k_{1}$ from Miller, et al.'s paper. In view of these difficulties a straightforward use of our equation 14 seems preferable.

\section{REFERENCES}

1. Ya. I. Frenkel, "Kinetic Theory of Liquids," Nauka, Leningrad, 1975, Chapter 7.

2. J. D. Hoffman, SPE Trans., 4, 315 (1964).

3. L. Mandelkern, "Crystallization of Polymers," MacGraw Hill Co., New York, N. Y., 1963.

4. D. Turnbull, in "Physics of Non-Crystalline Solids," J. A. Prins Ed., North-Holland, Amsterdam, $1965, \mathrm{p} 41$.

5. D. R. H. Jones, G. A. Chadwick, Phill. Mag., 22 291 (1970).

6. A. I. Rusanov, "Phase Equilibria and Surface Phenomena," Khimia, Leningrad, 1967.

7. Yu. V. Wulff, "Selected Papers on Crystal Physics and Crystallography," Gostekhteoretizdat, Moscow, 1952.

8. B. Wunderlich, "Macromolecular Physics," Vol. 1., Academic Press, New York, N. Y., 1973.

9. J. D. Hoffman and J. J. Weeks, J. Chem. Phys., 37, 1723 (1962). 
10. J. J. B. Blais and R. St. J. Manley, J. Macromol. Sci.-Phys., B1, 525 (1967).

11. J. G. Fatou, Eur. Polym. J., 7, 1057 (1971).

12. D. C. Bassett and R. Davitt, Polymer, 15, 721 (1974).

13. L. Mandelkern, N. L. Jain, and H. Kim, J. Polym. Sci., Part A-2, 6, 165 (1968).

14. T. Kawai, Kolloid Z. Z. Polym., 201, 15 (1965).

15. P. D. Calvert and D. R. Uhlmann, J. Appl. Phys., 43, 944 (1972).

16. I. V. Radchenko, "Molecular Physics," Nauka, Moscow, 1965.

17. V. K. Yatsimirskii, Teor. Eksp. Khim., 6, 704 (1970).

18. R. Hosemann and W. Wilke, Makromol. Chem., 112, 230 (1968).

19. H. ČačkoviČ, R. Hosemann, and W. Wilke, Kolloid Z. Z. Polym., 234, 1000 (1969).

20. I. R. Harrison and J. Runt, J. Polym. Sci., Polym. Phys. Ed., 14, 317 (1976).

21. I. R. Harrison, A. Keller, D. M. Sadler, and E. L. Thomas, Polymer, 17, 736 (1976).

22. J. Haase, R. Hosemann, and H. Čačkovič, Polymer, 18, 743 (1977).

23. A. I. Batchinskii, "Selected Papers," Izdatelistvo Akademia Nauk USSR Moscow, 1960.

24. F. B. Garner and S. Sugden, J. Chem. Soc., 1929, 1298.

25. R.-J. Roe, J. Phys. Chem., 72, 2013 (1968).

26. S. Wu, J. Macromol. Sci.-Revs. Macromol. Chem., C10, 1 (1974).

27. P. H. Geil, "Polymer Single Crystals," Interscience, New York, N. Y., 1963.

28. B. C. Edwards and P. J. Phillips, Polymer, 15, 351 (1975).

29. T. P. Hobin, J. Adhesion, 3, 237 (1972).

30. D. W. Van Krevelen (in collaboration with P. J. Hoftyzer)," Properties of Polymers," Elsevier, Amsterdam, 1972.

31. M. I. Bessonov and V. E. Smirnova, Vysokomol. Soedin., Ser. B, 13, 352 (1971).

32. Yu. S. Lipatov and V. P. Privalko, J. Macromol. Sci.-Phys., B7, 431 (1973).

33. V. P. Privalko, Yu. V. Pasechnik, L. I. Bezruk, and Yu. S. Lipatov, Vysokomol. Soedin., Ser. B, 13, 357 (1973).

34. V. P. Privalko, and Yu. S. Lipatov, Makromol. Chem., 175, 641 (1974).

35. F. Gornick and J. D. Hoffman, Ind. Eng. Chem., 58, 41 (1966).

36. V. P. Privalko, Polym. J., 7, 202 (1975).

37. J. Powers, J. D. Hoffman, J. J. Weeks, and F. A. Quinn, Jr., J. Res. Natl. Bur. Stand. 69A, 335 (1965).
38. F. C. Frank and C. Tosi, Proc. R. Soc., Ser. A, 263, 323 (1961).

39. V. P. Privalko, Preprint XXI-st International Symposium IUPAC on Macromolecules, Helsinki vol. 2, 1972, Abstract III-55, p 295.

40. V. P. Privalko, Vysokomol. Scedin., Ser, A, 15, 1905 (1973).

41. G. L. Slonimskii, A. A. Askadskii, and A. I. Kitaigorodskii, Vysokomol. Soedin., Ser. A, 12, 494 (1970).

42. A. I. Kitaigorodskii, "Molecular Crystals," Nauka, Moscow, 1971.

43. L. Mellillo and B. Wunderlich, Kolloid $Z$. $Z$. Polym., 250, 417 (1972).

44. R. G. Crystal, J. Polym. Sci., Part A-2, 1755 (1970).

45. R. G. Crystal, J. Polym. Sci., Part, A-2, 8, 2153 (1970).

46. A. Bondi, "Physical Properties of Molecular Crystals, Liquids and Glasses," Wiley, New York, N. Y., 1969.

47. A. A. Kudriyavtsev, "Chemistry and Technology of Selenium and Tellurium," Vysshaya Shkola, Moscow, 1961.

48. Yu. K. Godovskii, Vysokomol. Soedin. Part. A, 9, 2129 (1969).

49. F. J. Limbert and E. Baer, J. Polym. Sci. Part, A, 1, 3317 (1963).

50. T. Katz, J. Polym. Sci. Part, B, 8, 789 (1970).

51. J. Boon, C. Challa, and D. W. Van Krevelen, $J$. Polym. Sci., Part A-2, 6, 1791 (1968).

52. J. I. Lautirzen, Jr. and J. D. Hoffman, J. Appl. Phys., 44, 4340 (1973).

53. V.P. Privalko and Yu. S. Lipatov, Vysokomol. Soedin Ser., A, 18, 991 (1976).

54. V. P. Privalko, Yu. S. Lipatov, and A. P. Lobodina, J. Macromol. Sci.-Phys., B11, 441 (1975).

55. N. A. Smirnova, "Methods of Statistical Thermodynamics in Physical Chemistry," Vysshaya Shkola, Leningrad, 1973.

56. S. Glasston, K. Leidler, and H. Eyring, "Absolute Reactions Rate Theory," Interscience, New York, N. Y., 1940.

57. V. P. Privalko and Yu. S. Lipatov, Kolloid Z. Z. Polym., 251, 583 (1973).

58. A. Gandica and J. H. Magill, Polymer, 13, 595 (1972).

59. V. P. Privalko, unpublished work.

60. V. P. Privalko, Macromolecules, 6, 111 (1973).

61. V.P. Privalko, "Sintez i Fizitcheskie Khimiya Polimerov," Naukova Dumka, Kiev, No. 11, 1973, p 51.

62. V. P. Privalko and Yu. S. Lipatov, J. Polym. Sci., Polym. Phys. Ed., 14, 1725 (1976). 
63. C. Mancarella and E. Martuscelli, Polymer, 18, 1240 (1977).

64. A. Di Meo, G. Maglio, E. Martuscelli and R. Palumbo, Polymer, 17, 802 (1976).

65. D. Peterlin and E. Roeck1, J. Appl. Phys., 34, 102 (1963).

66. A. Peterlin, J. Appl. Phys., 35, 75 (1964).

67. J. D. Hoffman and J. J. Weeks, J. Chem. Phys., 42, 4301 (1965).

68. G. S. Fielding-Russel and R. E. Wetton, J. Polym. Sci., Part B, 5, 761 (1967).
69. D. R. Beech, C. Booth, D. V. Dodgson, and J. H. Hillier, J. Polym. Sci., Polym. Phys. Ed., 10, 1555 (1972).

70. M. Gordon and J. H. Hillier, Polymer, 6, 213 (1965).

71. P. J. Lemstra, A. J. Schouten and G. Challa, J. Polym. Sci., Polym. Phys. Ed., 12, 1505 (1974).

72. R. M. Gohil, K. C. Patel, and R. D. Patel, Polymer, 15, 402 (1974).

73. K. Ueberreiter, Kolloid Z. Z. Polym., 234, 1083 (1969). 